\title{
Modelagem matemática de um forno rotativo empregado na produção de clínquer
}

\section{(Mathematical modeling of a rotary kiln employed in the clínker production)}

\author{
D. C. Q. Rodrigues ${ }^{1}, A$. P. Soares $\mathrm{Jr}^{2}$, E. F. Costa Jr', A. O. S. Costa ${ }^{1}$ \\ ${ }^{I}$ Curso de Engenharia Química, Universidade Federal do Espírito Santo - CCA/UFES, Campus de Alegre, \\ Alto Universitário, Guararema, ES 29500-000 \\ ${ }^{2}$ Fábrica de Cimento Nassau, Cachoeiro de Itapemirim, ES \\ diuliacaroline@hotmail.com,atiliopeixoto@hotmail.com,eslyfcjr@yahoo.com.br,andreaosc@yahoo.com.br
}

\begin{abstract}
Resumo
Uma das principais etapas do processo de obtenção do cimento é a etapa de clinquerização, que ocorre dentro do forno rotativo. Em pleno funcionamento, o forno rotativo empregado na produção de cimento é alimentado basicamente de matéria-prima, combustível e ar. As vazões de saída são, basicamente, o clínquer e aos gases de combustão. Neste estudo, o processo de produção de clínquer por via seca é analisado, sendo a alimentação composta por $80 \%$ a $95 \%$ de calcário, $5 \%$ a $20 \%$ de argila e por pequenas quantidades de minério de ferro. O combustível empregado neste estudo é o carvão mineral. Os principais fenômenos envolvidos na obtenção do clínquer são a evaporação da água livre residual da matériaprima, a decomposição do carbonato de magnésio, a descarbonatação, a formação da fase líquida do $\mathrm{C}_{3} \mathrm{~A}$ e do $\mathrm{C}_{4} \mathrm{AF}$, a formação do silicato dicálcico e a formação do silicato tricálcico. O objetivo principal deste estudo é a proposta de um modelo matemático que descreve o processo de obtenção do clínquer no forno rotativo. O modelo, composto por equações algébricas, foi desenvolvido por meio de balanços de massa e energia do forno. A modelagem foi implementada em linguagem de programação, empregando-se um programa comercial. A condição operacional considerada representa uma condição média adotada na indústria. O modelo proposto se mostrou satisfatório para a descrição do processo de clinquerização, sendo especialmente útil na realização da análise da demanda energética do equipamento.

Palavras-chave: cimento Portland, forno rotativo, modelagem matemática, análise energética.
\end{abstract}

\begin{abstract}
An important stage of the cement production is the clinkerization process that occurs in the rotary kiln. Basically, the inputs of this equipment are the raw material, the fuel and the air. The output flows are basically the clinker and the combustion gases. In this study, the clinker production process by the dry process is analyzed. The considered feed of the rotary kiln is composed by $80 \%$ to $95 \%$ of lime, $5 \%$ to $20 \%$ clay and small quantities of iron ore. The fuel used in this study is coal. The main phenomena of the analyzed process are: water evaporation the residual free of the raw material, decomposition of magnesium carbonate, the carbon removal, the formation of the liquid phase $C_{3} A$ and $C_{4} A F$, the formation of dicalcium silicate and formation of tricalcium silicate. The main objective of this study is to propose a mathematical model that describes the production process of the clinker in the rotary kiln. The algebraic model proposed was developed considering the mass and the energy balances of the system. The model was solved using a commercial program (Mathcad). The operational condition considered represents a typical condition adopted in the industry. Theobtained results describesthe analyzed systemsatisfactorily, especially the energy demand of the equipment.
\end{abstract}

Keywords: Portland cement, rotary kiln, mathematical modeling, energy demand.

\section{INTRODUÇÃO}

O setor industrial de produção de cimento Portland, definição geral usada para cimentos fabricados a partir de matérias primas calcárias e argilosas, é tradicional no país [1]. Esta rota produtiva tem sido o foco de considerável atenção mundial por causa do alto consumo de energia e dos impactos ambientais causados pelo processo [2-10]. Sendo assim, as etapas envolvidas no processo produtivo devem ser cuidadosamente controladas, objetivando a operação ótima do sistema. Existem basicamente dois processos de produção de clínquer: por via úmida e por via seca [11]. Atualmente no Brasil toda a produção de cimento Portland é efetuada em fornos por via seca, uma vez que necessitam de 


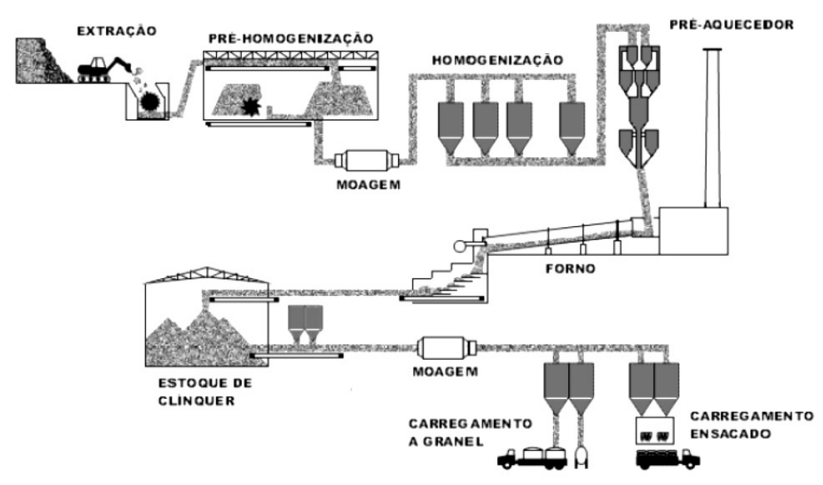

Figura 1: Representação esquemática do processo de produção de cimento Portland (adaptado [14]).

[Figure 1: Schematic representation of the Portland cement production (adapted [14]).]

menor quantidade de energia para a produção. Na produção por via seca, a mistura moída totalmente seca, alimenta o forno em forma de pó. De forma geral, o processo de produção de cimento Portland (Fig. 1), envolve a extração de matéria prima, moagem, homogeneização do calcário e argila, preparação e transporte do cru, a clinquerização (em fornos rotativos) e resfriamento dos produtos, adição de substâncias complementares e empacotamento [12, 13].

Uma das principais etapas do processo de obtenção do cimento é a etapa de clinquerização também chamada de sintetização, que ocorre dentro do forno rotativo, onde o clínquer é formado [15-17]. O clínquer é um material que se apresenta na forma de pelotas acinzentadas e foscas, de tamanho variado e com propriedades hidráulicas [18]. É composto basicamente por silicato dicálcio $\left(2 \mathrm{CaO}-\mathrm{SiO}_{2}\right)$, silicato tricálcio $\left(3 \mathrm{CaO}-\mathrm{SiO}_{2}\right)$, aluminato tricálcio $(3 \mathrm{CaO}$. $\left.\mathrm{Al}_{2} \mathrm{O}_{3}\right)$ e ferro-aluminato-tetracálcio (4CaO.Al2 $\mathrm{O}_{3} \cdot \mathrm{Fe} 2 \mathrm{O}_{3}$ ) [19-23]. A obtenção do clínquer ocorre entre $1450{ }^{\circ} \mathrm{C}$ e 1550 ${ }^{\circ} \mathrm{C}$ e envolve numerosos e complexos processos físicos e químicos. O desempenho do forno de cimento rotativo controla a qualidade do cimento e do desempenho geral da planta [24]. Os concretos atuais são compostos por uma mistura de cimento, água e agregados [25]. Os combustíveis convencionais usados no forno rotativo para a fabricação do clínquer são principalmente os carvões vegetais e minerais, o coque de petróleo e óleos pesados [13, 14]. Em pleno funcionamento, o forno rotativo empregado na produção de cimento é alimentado basicamente de matéria-prima,

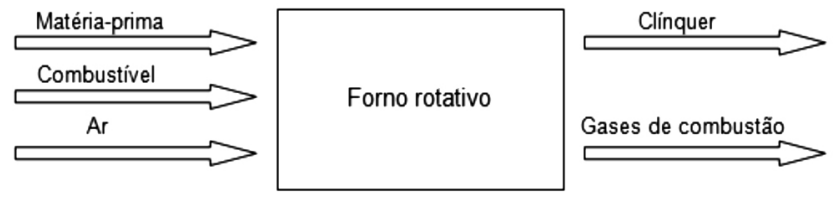

Figura 2: Representação esquemática das vazões de entrada e saída do forno rotativo.

[Figure 2: Schematic representation of the inputs and the outputs flows of the rotary kiln.]

combustível e ar. As vazões mássicas de saída são referentes ao clínquer e aos gases de combustão (Fig. 2). A composição da matéria-prima alimentada no forno rotativo, utilizado na indústria de cimento, depende especificamente de cada produção, assim como também a composição do clínquer obtido. Neste estudo destaca-se o processo de produção de clínquer por via seca, no qual a alimentação é composta por cerca de $80 \%$ a $95 \%$ de calcário $\left(\mathrm{CaCO}_{3}\right), 5 \%$ a $20 \%$ de argila $\left(\mathrm{SiO}_{2}, \mathrm{Al}_{2} \mathrm{O}_{3}, \mathrm{Fe}_{2} \mathrm{O}_{3}\right)$ e por pequenas quantidades de minério de ferro.

Ao ser alimentada a matéria-prima no sistema, iniciamse a evaporação da água livre residual e da água combinada das argilas e das reações características da obtenção do clínquer (Tabela I). O tempo médio de permanência é $~ 50$ $\min [26]$.

Cada uma das reações intermediárias de obtenção do clínquer ocorre sob determinada faixa de temperatura [29] (Tabela II). A temperaturas próximas de $1300{ }^{\circ} \mathrm{C}$, os compostos obtidos no processo de clinquerização $\mathrm{C}_{3} \mathrm{~A}$ $\left(3 \mathrm{CaO} \cdot \mathrm{Al}_{2} \mathrm{O}_{3}\right)$ e o $\mathrm{C}_{4} \mathrm{AF}\left(4 \mathrm{CaO} \cdot \mathrm{Al}_{2} \mathrm{O}_{3} \cdot \mathrm{Fe}_{2} \mathrm{O}_{3}\right)$ começam a se fundir, formando uma fase líquida localizada na interface dos grãos, que envolve os cristais de $\mathrm{C}_{2} \mathrm{~S}\left(2 \mathrm{CaO}\right.$. $\left.\mathrm{SiO}_{2}\right)$ já formados [30]. A temperatura final da pasta varia entre $50 \mathrm{e}$ $70^{\circ} \mathrm{C}$ e quanto mais rápido for este resfriamento, mais reativo será o cimento $[16,31]$. Na Tabela III são apresentados os calores das reações que ocorrem na produção do clínquer. Os gases provenientes da queima de combustível são retirados do sistema por um sistema de exaustão [14].

O objetivo geral deste trabalho consiste no desenvolvimento de um modelo matemático fenomenológico estático, através do emprego de balanços globais de massa e energia, para a descrição do processo de obtenção do clínquer em fornos rotativos empregados na rota de obtenção do cimento Portland. Os objetivos específicos consistem

Tabela I - Reações químicas do processo de obtenção do clínquer (adaptado [27]).

[Table I - Chemical reactions of the process of the clinker production (adapted [27]).]

\begin{tabular}{ll}
\hline Etapa do processo de clínquerização & Fenômenos envolvidos na clinquerização \\
\hline Decomposição do carbonato de magnésio & $\mathrm{MgCO}_{3}(\mathrm{~s}) \rightarrow \mathrm{MgO}(\mathrm{s})+\mathrm{CO}_{2}(\mathrm{~g})$ \\
Descarbonatação & $\mathrm{CaCO}_{3}(\mathrm{~s}) \rightarrow \mathrm{CaO}(\mathrm{s})+\mathrm{CO}_{2}(\mathrm{~g})$ \\
Formação da fase líquida & $3 \mathrm{CaO}(\mathrm{s})+\mathrm{Al}_{2} \mathrm{O}_{3}(\mathrm{~s}) \rightarrow 3 \mathrm{CaO}_{2} \mathrm{Al}_{2} \mathrm{O}_{3}(\mathrm{~s})\left(\mathrm{C}_{3} \mathrm{~A}\right)$ \\
Formação do silicato dicálcico & $4 \mathrm{CaO}(\mathrm{s})+\mathrm{Al}_{2} \mathrm{O}_{3}(\mathrm{~s})+\mathrm{Fe}_{2} \mathrm{O}_{3}(\mathrm{~s}) \rightarrow 4 \mathrm{CaO}_{2} \mathrm{Al}_{2} \mathrm{O}_{3} . \mathrm{Fe}_{2} \mathrm{O}_{3}(\mathrm{~s})\left(\mathrm{C}_{4} \mathrm{AF}\right)$ \\
Formação do silicato tricálcico & $2 \mathrm{CaO}(\mathrm{s})+\mathrm{SiO}_{2}(\mathrm{~s}) \rightarrow 2 \mathrm{CaO} . \mathrm{SiO}_{2}(\mathrm{~s})\left(\mathrm{C}_{2} \mathrm{~S}\right)$ \\
\hline
\end{tabular}


Tabela II - Temperaturas de obtenção das espécies químicas [27].

[Table II - The formation temperatures of the chemical species [27].]

\begin{tabular}{ll}
\hline Acima de $800{ }^{\circ} \mathrm{C}$ & Início do processo de fabricação de $\mathrm{CaO}$ \\
\hline Entre $800{ }^{\circ} \mathrm{C}$ e $1200{ }^{\circ} \mathrm{C}$ & Formação do $\mathrm{C}_{2} \mathrm{~S}$ \\
Entre $1095{ }^{\circ} \mathrm{C}$ e $1205^{\circ} \mathrm{C}$ & Formação do $\mathrm{C}_{3} \mathrm{~A}$ e $\mathrm{C}_{4} \mathrm{AF}$ \\
Entre $1260{ }^{\circ} \mathrm{C}$ e $1455^{\circ} \mathrm{C}$ & $\begin{array}{l}\text { Formação do } \mathrm{C}_{3} \mathrm{~S} \text { a partir do } \mathrm{C}_{2} \mathrm{~S} \text { com quase extinção da cal livre }(\mathrm{CaO}) \\
\text { Cristalização da fase líquida do } \mathrm{C}_{3} \mathrm{~A} \text { e do } \mathrm{C}_{4} \mathrm{AF} .\end{array}$ \\
Entre $1455^{\circ} \mathrm{C}$ e $1300{ }^{\circ} \mathrm{C}$ & $\begin{array}{l}\text { Os silicatos } \mathrm{C}_{2} \mathrm{~S} \text { e } \mathrm{C}_{3} \mathrm{~S} \text { praticamente não sofrem alterações tanto na forma como na } \\
\text { composição }\end{array}$ \\
\hline
\end{tabular}

Tabela III - Calores padrão de reação para o processo de obtenção de clínquer [27, 28]. [Table III - The standard heat of reaction to the clinker production [27, 28].]

\begin{tabular}{|c|c|c|c|}
\hline \multicolumn{2}{|c|}{ Reações } & \multicolumn{2}{|c|}{ Calor de Reação (a $\left.20^{\circ} \mathrm{C}\right)(\mathrm{kJ} / \mathrm{kg})$} \\
\hline$\overline{\mathrm{R} 1}$ & $\mathrm{CaCO}_{3} \longleftrightarrow \mathrm{CaO}+\mathrm{CO}_{2}$ & $\overline{D R} 1$ & +1766 \\
\hline R1a & $2 \mathrm{CaO}+\mathrm{SiO}_{2} \rightarrow 2 \mathrm{CaO} . \mathrm{SiO}_{2}$ & DR1a & -717 \\
\hline $\mathrm{R} 1 \mathrm{~b}$ & $3 \mathrm{CaO}+\mathrm{SiO}_{2}^{2} \rightarrow 3 \mathrm{CaO} \cdot \mathrm{SiO}_{2}^{2}$ & DR1b & -528 \\
\hline $\mathrm{R} 1 \mathrm{c}$ & $\mathrm{CaO}+\mathrm{Al}_{2} \mathrm{O}_{3}^{2} \rightarrow \mathrm{CaO} \cdot \mathrm{Al}_{2} \mathrm{O}_{3}{ }^{2}$ & DR1c & -309 \\
\hline R1d & $12 \mathrm{CaO}+7 \mathrm{Al}_{2} \mathrm{O}_{3} \rightarrow 12 \mathrm{CaO} .7 \mathrm{Al}_{2} \mathrm{O}_{3}$ & DR1d & -169 \\
\hline R1e & $3 \mathrm{CaO}+\mathrm{Al}_{2} \mathrm{O}_{3} \rightarrow 3 \mathrm{CaO} \cdot \mathrm{Al}_{2} \mathrm{O}_{3}$ & DR1e & -15 \\
\hline R1f & $4 \mathrm{CaO}+\mathrm{Al}_{2} \mathrm{O}_{3}+\mathrm{Fe}_{2} \mathrm{O}_{3} \rightarrow 4 \mathrm{CaO} \cdot \mathrm{Al}_{2} \mathrm{O}_{3} \cdot \mathrm{Fe}_{2} \mathrm{O}_{3}$ & DR1f & -717 \\
\hline $\mathrm{R} 2$ & $\mathrm{MgCO}_{3} \rightarrow \mathrm{MgO}+\mathrm{CO}_{2}$ & DR2 & -1188 \\
\hline R3 & $\mathrm{C}+\mathrm{O}_{2} \rightarrow \mathrm{CO}_{2}$ & DR3 & $-393,5$ \\
\hline
\end{tabular}

em calcular a temperatura final de obtenção do clínquer, considerando a perda de energia por meio da radiação, e calcular a temperatura adiabática do processo. Além disso, utilizando dados industriais, adaptar o modelo á realidade e determinar a temperatura de alimentação de ar no forno.

\section{MATERIAIS E MÉTODOS}

Como não foram encontradas em literatura aberta as equações de capacidade calorífica dos compostos $\mathrm{C}_{3} \mathrm{~A} 7$, $\mathrm{C}_{12} \mathrm{~A}, \mathrm{CA}, \mathrm{C}_{4} \mathrm{AF}, \mathrm{C}_{2} \mathrm{~S}$ e $\mathrm{C}_{3} \mathrm{~S}$, a metodologia empregada

Tabela IV - Capacidade calorífica [32, 33].

[Table IV - Heat capacity [32, 33].]

\begin{tabular}{ll}
\hline Espécies Químicas & Capacidade calorífica $(\mathrm{Cp})(\mathrm{J} / \mathrm{mol} . \mathrm{K})$ \\
\hline $\mathrm{CaCO}_{3}$ & $82,2624+0,0497 \times \mathrm{T}-(1285768) / \mathrm{T}^{2}$ \\
$\mathrm{CaO}$ & $41,8+0,0202 \times \mathrm{T}-(451440) / \mathrm{T}^{2}$ \\
$\mathrm{MgCO}$ & 70,642 \\
$\mathrm{MgO}$ & $45,3948+0,005003 \times \mathrm{T}-(872366) / \mathrm{T}^{2}$ \\
$\mathrm{SiO}$ & $53,504+0,0186846 \times \mathrm{x}-(1262360) / \mathrm{T}^{2}$ \\
$\mathrm{Al}_{2} \mathrm{O}_{3}$ & $92,2944+0,03749878 \times \mathrm{xT}-(218405) / \mathrm{T}^{2}$ \\
$\mathrm{Fe}_{2} \mathrm{O}_{3}$ & $103,3296+0,0670472 \times \mathrm{T}-(1769812) / \mathrm{T}^{2}$ \\
$\mathrm{CO}_{2}$ & $43,2212+0,0114532 \times \mathrm{T}-(817190) / \mathrm{T}^{2}$ \\
$\mathrm{H}_{2} \mathrm{O}(\mathrm{l})$ & $72,431568+0,0103925 \times \mathrm{x}-(1496520) / \mathrm{T}^{2}$ \\
$\mathrm{H}_{2} \mathrm{O}(\mathrm{g})$ & $28.84958+0,0120553 \times \mathrm{T}-1005994 \times \mathrm{T}^{2}$ \\
$3 \mathrm{CaO} . \mathrm{SiO}_{2}$ & $44,884+0,01982367 \times \mathrm{x}-(665117,42) / \mathrm{T}^{2}$ \\
$2 \mathrm{CaO} . \mathrm{SiO}_{2}$ & $45,8964+0,01968989 \times \mathrm{xT}-(735262) / \mathrm{T}^{2}$ \\
$12 \mathrm{CaO} .7 \mathrm{Al}_{2} \mathrm{O}_{3}$ & $67,8046+0,02912206 \times \mathrm{T}-(1343732,06) / \mathrm{T}^{2}$ \\
$\mathrm{CaO} \cdot \mathrm{Al}_{2} \mathrm{O}_{3}$ & $73,6934+0,031079972 \times \mathrm{T}-(1555796) / \mathrm{T}^{2}$ \\
$3 \mathrm{CaO} . \mathrm{Al}_{2} \mathrm{O}_{3}$ & $60,987872+0,02678962 \times \mathrm{T}-(1109831,8) / \mathrm{T}^{2}$ \\
$4 \mathrm{CaO} . \mathrm{Al}_{2} \mathrm{O}_{3} \cdot \mathrm{Fe}_{2} \mathrm{O}_{3}$ & $72,708592+0,039229 \times \mathrm{T}-(1250350,86) / \mathrm{T}^{2}$ \\
$\mathrm{C}(\mathrm{grafite})$ & $14,724094+0,006410094 \times \mathrm{T}-(720823,8) / \mathrm{T}^{2}$ \\
$\mathrm{O}_{2}$ & $30,254646+0,004206884 \times \mathrm{x}-(188727,8) / \mathrm{T}^{2}$ \\
$\mathrm{~N}_{2}$ & $27,26992+0,004930202 \times \mathrm{T}-(33256) / \mathrm{T}^{2}$ \\
\hline
\end{tabular}


consistiu inicialmente na estimativa destes parâmetros. Considerando a composição estequiométrica destas espécies, foram propostas relações mássicas baseadas nas capacidades caloríficas dos compostos químicos mais simples, que se encontram disponíveis na literatura. As equações das capacidades caloríficas utilizadas para os compostos envolvidos no processo de sintetização são apresentados na Tabela IV.

O modelo matemático fenomenológico proposto foi desenvolvido a partir de balanços estáticos de massa e energia do forno. As principais relações matemáticas que compõem o modelo e o fluxograma empregado em sua resolução são apresentados na Fig. 3. A solução numérica foi obtida empregando-se o programa comercial Mathcad.

\section{RESULTADOS E DISCUSSÃO}

Partindo de adaptações do trabalho [5], foram adotados valores das variáveis de entrada do modelo (entrada V). Tradicionalmente, a temperatura média de saída do

\section{Cálculo}

Composição mássica de saídas de todas as substâncias envolvidas no processo

\section{$\downarrow$}

Dados de entrada:

Composição de entrada da matéria prima: m1 Composição mássica do $\mathrm{Al}_{2} \mathrm{O}_{3}(\mathrm{p} / \mathrm{p})$ : xa

Massa de combustível: mCExcesso de ar alimentado: exar

Composição mássica de calcário (p/p): xcalTemperatura de alimentação da matéria prima: T0

Composição mássica das argilas (p/p): xmTemperatura de alimentação do combustível

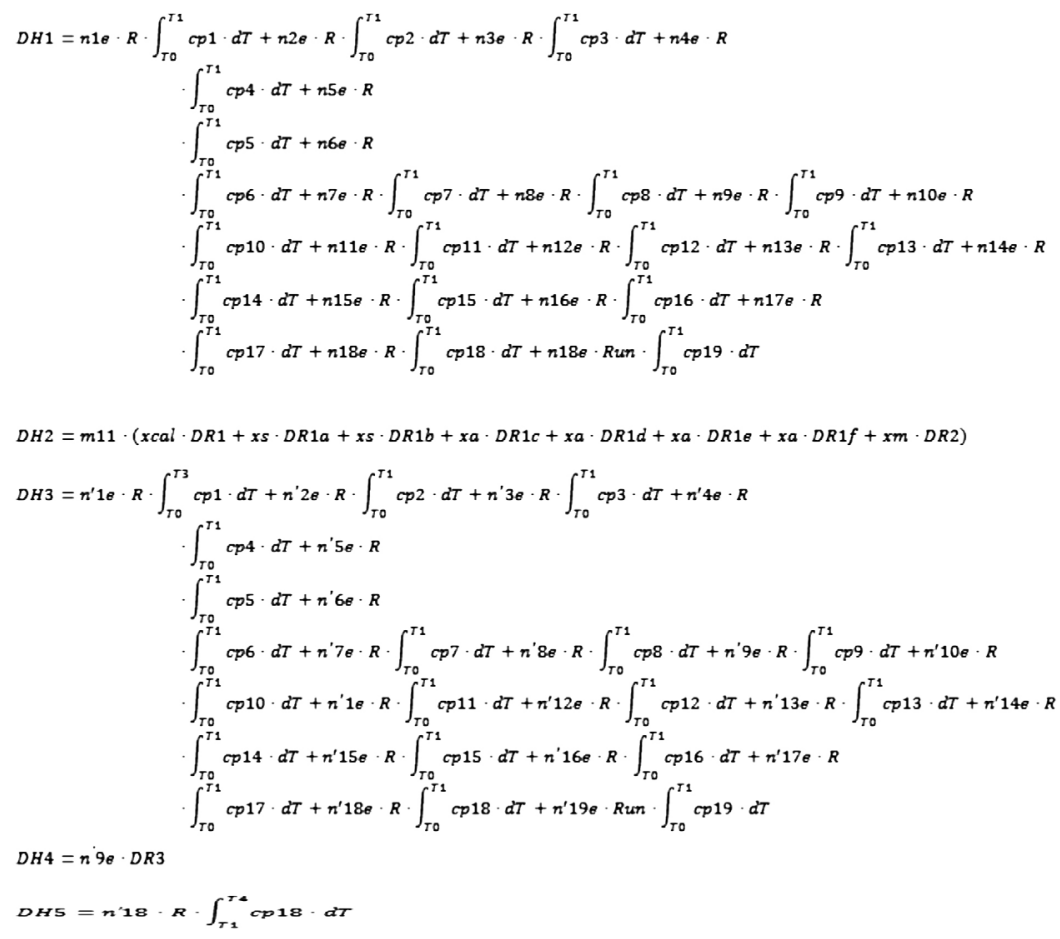

Soma de todos os DH calculados

$\downarrow$

Temperatura adiabática de obtenção do clínquer: Tfa $(\mathrm{K})$ 
Tabela $\mathrm{V}$ - Dados de entrada e saída do modelo matemático com perdas de energia devido a radiação. [Table $V$ - The inputs and the outputs of the mathematical model considering radiation losses.]

\begin{tabular}{ll}
\hline Entrada & Saída \\
\hline Matéria prima: 2000 toneladas/dia & Composição mássica do $\mathrm{C}_{2} \mathrm{~S}(\mathrm{p} / \mathrm{p}): 0,1626$ \\
Massa de combustível: 170 toneladas/dia & Composição mássica do $\mathrm{C}_{3} \mathrm{~S}(\mathrm{p} / \mathrm{p}): 0,5449$ \\
Composição mássica de calcário $(\mathrm{p} / \mathrm{p}): 0,6859$ & Composição mássica do $\mathrm{CA}(\mathrm{p} / \mathrm{p}): 0,0571$ \\
Composição mássica do $\mathrm{MgCO}_{3}(\mathrm{p} / \mathrm{p}): 0,0258$ & Composição mássica do $\mathrm{C}_{12} \mathrm{~A}(\mathrm{p} / \mathrm{p}): 0,0513$ \\
Composição mássica do $\mathrm{SiO}_{2}(\mathrm{p} / \mathrm{p}): 0,2071$ & Composição mássica do $\mathrm{C}_{3} \mathrm{~A}(\mathrm{p} / \mathrm{p}): 0,0940$ \\
Composição mássica do $\mathrm{Al}_{2} \mathrm{O}_{3}(\mathrm{p} / \mathrm{p}): 0,0484$ & Composição mássica do $\mathrm{C}_{4} \mathrm{AF}(\mathrm{p} / \mathrm{p}): 0,0897$ \\
Composição mássica do $\mathrm{Fe}_{2} \mathrm{O}_{3}(\mathrm{p} / \mathrm{p}): 0,0278$ & \\
Composição mássica do $\mathrm{H}_{2} \mathrm{O}(\mathrm{p} / \mathrm{p}): 0,005$ & Quantidade de calor perdido por radiação: $8510 \mathrm{~W}$ \\
$\begin{array}{l}\text { Excesso de ar alimentado: } 12 \% \\
\text { Temperatura de alimentação da matéria prima: } 50{ }^{\circ} \mathrm{C}\end{array}$ & Temperatura de saída do clínquer: $1255,85{ }^{\circ} \mathrm{C}$ \\
Temperatura de alimentação do combustível: $25^{\circ} \mathrm{C}$ & \\
Temperatura de alimentação de ar: $680{ }^{\circ} \mathrm{C}$ & \\
\hline
\end{tabular}

clínquer é de 1200 a $1300{ }^{\circ} \mathrm{C}[22,30]$. De acordo com dados industriais fornecidos pelo profissional da fábrica, para um forno $2.000 \mathrm{t} /$ dia de matéria-prima necessitase de cerca de 170 t/dia de combustível, e a taxa usual de excesso de arno processo de clinquerização é de 8 a $15 \%$. O ar alimentado no forno é pré-aquecido com o ar

Tabela VI - Composição química do clínquer (adaptado de $[8,10,30,34])$.

[Table VI - The clinker chemical composition (adapted [8, 10, 30, 34]).]

\begin{tabular}{lc}
\hline \multicolumn{2}{c}{$\begin{array}{c}\text { Composição do clinquer } \\
(\mathrm{p} / \mathrm{p})\end{array}$} \\
\hline $\mathrm{C}_{2} \mathrm{~S}$ & 0,15 \\
$\mathrm{C}_{3} \mathrm{~S}$ & 0,56 \\
$\mathrm{CA}$ & 0,055 \\
$\mathrm{C}_{12} \mathrm{~A}_{7}$ & 0,045 \\
$\mathrm{C}_{3} \mathrm{~A}$ & 0,09 \\
$\mathrm{C}_{4} \mathrm{AF}$ & 0,1 \\
\hline
\end{tabular}

quente resultante do processo de resfriamento do clínquer [27]. Comparando os valores simulados (saída, Tabela V) com os valores encontrados em literatura (Tabela VI), é possível verificar a adequação da metodologia proposta para o cálculo das composições de saída do forno.

Em um segundo momento, foi feita a simulação da temperatura adiabática do forno. O valor simulado apresentado na Tabela VII é consideravelmente maior do que o encontrado para a temperatura final de obtenção do clínquer, quando se leva em conta a perda de calor por radiação (Tabela VI). Isso já era esperado uma vez que a perda de calor por radiação diminiu a eficiência energética do processo.

\section{CONCLUSÕES}

Foi desenvolvido um modelo matemático capaz de descrever o processo de obtenção do clínquer no forno rotativo empregado na produção de cimento Portland. A condição operacional considerada para a elaboração do modelo matemático representa uma condição média adotada na indústria. A análise dos resultados obtidos

Tabela VII - Dados de entrada e saída do modelo matemático considerando processo adiabático.

[Table VII - The inputs and the outputs of the mathematical model considering adiabatic process.]

\begin{tabular}{ll}
\hline Entrada & Saída \\
\hline Matéria prima: 2000 toneladas/dia & Composição mássica do $\mathrm{C}_{2} \mathrm{~S}(\mathrm{p} / \mathrm{p}): 0,1626$ \\
Massa de combustível: 170 toneladas/dia & Composição mássica do $\mathrm{C}_{3} \mathrm{~S}(\mathrm{p} / \mathrm{p}): 0,5449$ \\
Composição mássica de calcário $(\mathrm{p} / \mathrm{p}): 0,6859$ & Composição mássica do $\mathrm{CA}(\mathrm{p} / \mathrm{p}): 0,0571$ \\
Composição mássica do $\mathrm{MgCO}_{3}: 0,0258$ & Composição mássica do $\mathrm{C}_{12} \mathrm{~A}_{7}(\mathrm{p} / \mathrm{p}): 0,0513$ \\
Composição mássica do $\mathrm{SiO}_{2}(\mathrm{p} / \mathrm{p}): 0,2071$ & Composição mássica do $\mathrm{C}_{3} \mathrm{~A}(\mathrm{p} / \mathrm{p}): 0,0940$ \\
Composição mássica do $\mathrm{Al}_{2} \mathrm{O}_{3}(\mathrm{p} / \mathrm{p}): 0,0484$ & Composição mássica do $\mathrm{C}_{4} \mathrm{AF}(\mathrm{p} / \mathrm{p}): 0,0897$ \\
Composição mássica do $\mathrm{Fe}_{2} \mathrm{O}_{3}(\mathrm{p} / \mathrm{p}): 0,0278$ & \\
Composição mássica do $\mathrm{H}_{2} \mathrm{O}(\mathrm{p} / \mathrm{p}): 0,005$ & Temperatura de saída do clínquer (temperatura \\
Excesso de ar alimentado: $12 \%$ & adiabática): $1474,85{ }^{\circ} \mathrm{C}$ \\
Temperatura de alimentação da matéria prima: $50{ }^{\circ} \mathrm{C}$ & \\
Temperatura de alimentação do combustível: $25^{\circ} \mathrm{C}$ & \\
Temperatura de alimentação de ar: $680{ }^{\circ} \mathrm{C}$ & \\
\hline
\end{tabular}


indica que a metodologia proposta é adequada para o cálculo da composição de saída dos compostos. Foi possível ainda calcular a temperatura final de obtenção do clínquer considerando a perda de calor por radiação. A temperaura adiabática do forno também foi obtida e seu valor representa um limite máximo para a temperatura no interior do equipamento. $\mathrm{O}$ modelo matemático proposto neste trabalho não requer recursos computacionais avançados para ser resolvido e pode ser empregado na busca de novas condições operacionais para o equipamento. Diante da preocupação crescente do setor produtivo com o uso eficiente de energia, tais análises são fundamentais quando se deseja otimizar o consumo energético industrial.

\section{AGRADECIMENTO}

Os autores agradecem o apoio financeiro concedido pela UFES (Universidade Federal do Espírito Santo) através do Programa Institucional de Iniciação Científica (PIIC).

\section{NOMENCLATURA}

\begin{tabular}{|c|c|c|}
\hline p1 & apacidade calorífica do $\mathrm{CaCO}_{3}$ & {$[\mathrm{~J} /(\mathrm{mol} . \mathrm{K})$} \\
\hline $\mathrm{p} 2$ & Capacidade calorífica do $\mathrm{CaO}$ & {$[\mathrm{J} /(\mathrm{mol}$} \\
\hline 3 & Capacidade calorífica do $\mathrm{CO}_{2}$ & {$\left[L^{0}\right.$} \\
\hline 4 & Capacidade calorífica do $\mathrm{MgCO}_{3}$ & {$[\mathrm{~J} /(\mathrm{mol}$} \\
\hline & Capacidade calorífica do $\mathrm{MgO}$ & {$[\mathrm{J} /(\mathrm{mc}$} \\
\hline & Capacidade calorífica do $\mathrm{SiO}_{2}$ & \\
\hline 7 & Capacidade calorífica do $\mathrm{Al}_{2} \mathrm{O}_{3}$ & {$[\mathrm{~J} /(\mathrm{mol}$} \\
\hline 8 & Capacidade calorífica do $\mathrm{Fe}_{2} \mathrm{O}_{3}$ & {$[\mathrm{~J} /(\mathrm{mo}$} \\
\hline p9 & Capacidade calorífica do C & {$[\mathrm{J}$} \\
\hline ep 10 & Capacidade calorífica do $\mathrm{O}_{2}$ & {$[\mathrm{~J} /(\mathrm{mol}$.} \\
\hline 11 & Capacidade calorífica do $\mathrm{N}_{2}$ & {$[\mathrm{~J} /(\mathrm{mo}$} \\
\hline 12 & Capacidade calorífica do $2 \mathrm{CaO} \cdot \mathrm{SiO}_{2}$ & {$[\mathrm{~J} /(\mathrm{mol}$} \\
\hline 13 & Capacidade calorífica do $3 \mathrm{CaO} \cdot \mathrm{SiO}_{2}$ & {$[\mathrm{~J} /(\mathrm{mol}$} \\
\hline 14 & Capacidade calorífica do $\mathrm{CaO} \cdot \mathrm{Al}_{2} \mathrm{O}_{3}$ & {$[\mathrm{~J} /(\mathrm{mo}$} \\
\hline 15 & Capacidade calorífica do $12 \mathrm{CaO} .7 \mathrm{Al}_{2} \mathrm{O}_{3}$ & {$[\mathrm{~J} /(\mathrm{mol}$.} \\
\hline 16 & Capacidade calorífica do $3 \mathrm{CaO} \cdot \mathrm{Al}_{2} \mathrm{O}_{3}$ & {$[\mathrm{~J} /(\mathrm{mol}$.} \\
\hline $1^{\prime}$ & $\begin{array}{l}\text { Capacidade Calorífica do } 4 \mathrm{CaO} \cdot \mathrm{Al}_{2} \mathrm{O}_{3} \text {. } \\
\mathrm{Fe}_{2} \mathrm{O}_{3}\end{array}$ & {$[\mathrm{~J} /(\mathrm{mo}$} \\
\hline & Capacidade calorífica do $\mathrm{H}_{2} \mathrm{O}(\mathrm{l})$ & {$[\mathrm{J} /(\mathrm{mol} . \mathrm{H}$} \\
\hline 19 & Capacidade calorífica da $\mathrm{H}_{2} \mathrm{O}$ & {$[\mathrm{J} /(\mathrm{mol}$.} \\
\hline $\mathrm{H}$ & $\begin{array}{l}\text { Calor sensível para levar os reagentes } \\
\text { da temperatura de entrada até a } \\
\text { temperatura de decomposição do } \\
\mathrm{CaCO}_{3} \text { e do } \mathrm{MgCO}_{3}\end{array}$ & {$[\mathrm{~J} /(\mathrm{mol}$} \\
\hline
\end{tabular}

$\mathrm{DH}_{2} \quad$ Soma do calor de reação de decomposição do $\mathrm{MgCO}_{3}$ e calor de formação do $\mathrm{C}_{3} \mathrm{~A}, \mathrm{C}_{4} \mathrm{~A}, \mathrm{C}_{4} \mathrm{AF}, \mathrm{C}_{2} \mathrm{~S}, \mathrm{C}_{3} \mathrm{~S}$

$\mathrm{DH}_{3} \quad$ Calor sensível para levar os produtos $\mathrm{C}_{3} \mathrm{~A}, \mathrm{C}_{4} \mathrm{~A}, \mathrm{C}_{4} \mathrm{AF}, \mathrm{C}_{2} \mathrm{~S}, \mathrm{C}_{3} \mathrm{~S}, \mathrm{CO}_{3}, \mathrm{MgO}$ e $\mathrm{CO}_{3}$ para a temperatura de queima do combustível.

$[\mathrm{J} /(\mathrm{mol} . \mathrm{K})]$
$\mathrm{DH}_{4} \quad$ Calor de reação de combustão

$[\mathrm{J} /(\mathrm{mol} . \mathrm{K})]$

$\mathrm{DH}_{5} \quad$ Calor sensível necessário para levar a água da temperatura ambiente para a temperatura de evaporação.

$\mathrm{DH}_{6} \quad$ Calor latente de evaporação da água

$\mathrm{DH}_{7} \quad$ Calor necessário para levar o vapor de água da temperatura de evaporação para a temperatura de saída do clinquer

$\mathrm{DH}_{8} \quad$ Calor de fusão do $\mathrm{C}_{3} \mathrm{~A}$

$[\mathrm{J} /(\mathrm{mol} . \mathrm{k})]$

$\mathrm{DH}_{9} \quad$ Calor de fusão do $\mathrm{C}_{4} \mathrm{AF}$

$\mathrm{DH}_{10} \quad$ Calor sensível para levar todos os produtos gerados no processo para temperatura final de obtenção doclinquer

DR4 Calor de vaporização da água

$[\mathrm{J} /(\mathrm{mol} . \mathrm{K})]$

$[\mathrm{J} /(\mathrm{mol} . \mathrm{K})]$

$[\mathrm{J} /(\mathrm{mol} . \mathrm{K})]$

Calor de fusão do $\mathrm{C}_{3} \mathrm{~A}$

DR6 Calor de fusão do $\mathrm{C}_{4} \mathrm{AF}$

[J/(mol.k)]

[J/(mol.K)]

n1e

número de mols de $\mathrm{CaCO}_{3}$ que entram no forno

$\mathrm{n} 2 \mathrm{e}$

número de mols de $\mathrm{CaO}$ que entram no forno

n3e

número de mols de $\mathrm{CO}_{2}$ que entram no forno

$\mathrm{n} 4 \mathrm{e}$

número de mols de $\mathrm{MgCO}$ que entram no forno

$\mathrm{n} 5 \mathrm{e}$

número de mols de $\mathrm{CaO}$ que entram no forno

n6e

número forno

n7e

número forno

n8e

número de mols de $\mathrm{Fe}_{2} \mathrm{O}_{3}$ que entram no forno

n9e

número c forno

n10e

número d

n11e

forno

número de mols de $\mathrm{N}_{2}$ que entram no

forno

$\mathrm{n} 12 \mathrm{e}$

número de mols de $2 \mathrm{CaO} . \mathrm{SiO}_{2}$ que entram no forno

n13e

número de mols

entram no forno

$\mathrm{n} 14 \mathrm{e}$

número de mols

entram no forno

n15e

número de mols entram no forno

n16e

número de mols

de $3 \mathrm{CaO} . \mathrm{SiO}_{2}$ que

de $\mathrm{CaO} \cdot \mathrm{Al}_{2} \mathrm{O}_{3}$ que

$12 \mathrm{CaO} .7 \mathrm{Al}_{2} \mathrm{O}_{3}$ que

$.7 \mathrm{Al}_{2} \mathrm{O}_{3}$ que

[J/(mol.K)]

$[\mathrm{J} /(\mathrm{mol} . \mathrm{K})]$

[J/(mol.K)]

[mol]

[mol]

[mol]

[mol]

[mol]

[mol]

[mol]

[mol]

[mol]

[mol]

[mol]

[mol]

[mol]

[mol]

[mol]

entram no forno

n17e

número de mols de

que entram no forno

n18e

número de

n19e

no forno

número de mols de $\mathrm{H}_{2} \mathrm{O}(\mathrm{v})$ que entram no forno

n'1e

número de mols de $\mathrm{CaCO}_{3}$ presentes

quando ocorre a reação de combustão

n’2e número de mols de $\mathrm{CaO}$ presentes

quando ocorre a reação de combustão [mol]

[mol]

[mol]

[mol]

[mol]

[mol] 
n’3e número de mols de $\mathrm{CO}_{2}$ presentes quando ocorre a reação de combustão

n'4e número de mols de $\mathrm{MgCO}$ presentes quando ocorre a reação de combustão

n'5e número de mols de $\mathrm{CaO}$ presentes quando ocorre a reação de combustão

n'6e número de mols de $\mathrm{SiO}_{2}$ presentes quando ocorre a reação de combustão

n'7e número de mols de $\mathrm{Al}_{2} \mathrm{O}_{3}$ presentes quando ocorre a reação de combustão

n'8e número de mols de $\mathrm{Fe}_{2} \mathrm{O}_{3}$ presentes quando ocorre a reação de combustão

n'9e número de mols de $\mathrm{C}$ presentes quando [mol] ocorre a reação de combustão

n'10e número de mols de $\mathrm{O}_{2}$ presentes [mol] quando ocorre a reação de combustão

n'11e número de mols de $\mathrm{N}_{2}$ presentes quando [mol] ocorre a reação de combustão

n'12e número de mols de $2 \mathrm{CaO} \cdot \mathrm{SiO}_{2}$ presentes quando ocorre a reação de combustão

n'13e número de mols de $\mathrm{N}_{2}$ presentes quando ocorre a reaçã̃o de combustão

n'14e número de mols de $\mathrm{CaO} \cdot \mathrm{Al}_{2} \mathrm{O}_{3}$ presentes quando ocorre a reação de combustão

n'15e número de mols de $12 \mathrm{CaO} .7 \mathrm{Al}_{2} \mathrm{O}_{3}$ presentes quando ocorre a reaçã̃o de combustão

n'16e número de mols de $3 \mathrm{CaO} . \mathrm{Al}_{2} \mathrm{O}_{3}$ presentes quando ocorre a reação de combustão

n'17e número de mols de $4 \mathrm{CaO} \cdot \mathrm{Al}_{2} \mathrm{O}_{3} \cdot \mathrm{Fe}_{2} \mathrm{O}_{3}$ presentes quando ocorre a reação de combustão

n'18e número de mols de $\mathrm{H}_{2} \mathrm{O}(\mathrm{l})$ presentes quando ocorre a reação de combustão

n'19e número de mols de $\mathrm{H}_{2} \mathrm{O}$ (v) presentes quando ocorre a reação de combustão

$\mathrm{R} \quad$ Constante universal dos gases

[mol]

[mol]

[mol]

[mol]

[mol]

[mol]

[mol]

[mol]

T0 Temperatura de alimentação da matéria $\mathrm{K}$ prima

Tf Temperatura de saída do clinquer $\quad \mathrm{K}$

T1 Temperatura em que ocorre a reação de $\mathrm{K}$ formação do óxido de cálcio

T3 Temperatura em que ocorre a $\quad \mathrm{K}$ combustão do carvão

T4 Temperatura em que ocorre a vaporização do $\mathrm{H}_{2} \mathrm{O}$

y5 Fração molar de $\mathrm{CaO}$ que segue a $\mathrm{K}$ equação R1e

y6 Fração molar de $\mathrm{CaO}$ que segue a $\quad \mathrm{K}$ equação R1f

\section{REFERÊNCIAS}

[1] ABCP, Associação Brasileira de Cimento Portland, http://www.abcp.org.br/conteudo/basico-sobre-cimento/ historia/uma-breve-historia do-cimento-portland, acesso em 14/11/2011.

[2] E. M. Gartner, D. E. Macphee, A physico-chemical basis for novel cementitious binders, Cement Concr. Res. 41 (2011) 736-749.

[3] K. S. B. Mujumdara, K. V. Ganesha, S. B. Kulkarnia, V. V. Ranade, Rotary Cement Kiln Simulator (RoCKS): Integrated modeling of pre-heater, calciner, kiln and clinker cooler, Chem. Eng. Sci. 62, 9 (2007) 2590-2607.

[4] Z. Utlu, Z. Sogut, A. Hepbasli, Z. Oktay, Energy and exergy analyses of a raw mill in a cement production, Appl. Thermal Eng. 26, 17-18 (2006) 2479-2489.

[5] T. Engin, V. Ari, Energy auditing and recovery for dry type cement rotary kiln systems - a case study - Departamento de engenharia mecânica, Universidade de Sakarya, Energy conversion e management (2004).

[7] E. Worrell, N. Martin, L. Price, Potentials for energy efficiency improvement in the US cement industry, Energy (1999) 26.

[8] E. A. Mastorakos, A. Massias, C. D. Tsakiroglou, D. A. Goussis, V. N. Burganos, A. C. Payatakes, CFD predictions for cement kilns including flame modelling, heat transfer and clinker chemistry, Appl. Mathematical Modeling (1998) 22.

[9] A. M. M. Santi, O emprego de resíduos como combustíveis complementares na produção de cimento na perspectiva da energia, da sociedade e do meio ambiente, Diss. Mestrado, Planejamento de Sistemas Energéticos, Faculdade de Engenharia Mecânica, UNICAMP (1997).

[10] K. E. Peray, The Rotary Cement Kiln, Chem. Publishing Co. (1986) 388.

[11] R. J. Silva, Análise Energética de Plantas de Produção de Cimento Portland, Tese Dr., Engenharia Mecânica, UNICAMP (1994) 242.

[12] C. Chen, G. Habert, Y. Bouzidi, A. Jullien, Environmental impact of cement production: detail of the different processes and cement plant variability evaluation, J. Cleaner Production (2010) 478-485.

[J/(mol.K)] [13]A.C.S.M.Bernardo,R.C.Carpio,R.J.Silva,Otimização Multi-Objetivos da Produção de Cimento Portland com CoProcessamento e Adição de Mineralizadores, Universidade Federal de Itajubá - UNIFEI, Itajubá, MG, Nono Simpósio de Mecânica Computacional (2010) 10.

[14] M. L. G. Renó, Uso de Técnicas de Otimização Robusta Multi-Objetivos na Produção de Cimento, Diss. Mestrado, Engenharia Mecânica, Universidade Federal de Itajubá, MG (2007) 141.

[15] R. Saidur, M. S. Hossain, M. R. Islam, H. Fayaz, H. A. Mohammed, A review on kiln system modeling, Energy (2007) 2487-2500.

[16] M. C. C. Silva, Relações entre microestrutura, composição, resistência à ruptura e moabilidade de clínqueres de cimento Portland, Diss. Mestrado, Engenharia Metalúrgica e de Materiais, COPPE/UFRJ, Rio de Janeiro, RJ (2007).

[17] A. A. Boateng, P. V. Barr, A thermal model for the rotary kiln including heat transfer within the bed, Department of Metals and Materials Engineering, The University of British Columbia,Vancouver, B.C., Canada V6T 124. Pergamon (1995) 2131-2147. 
[18] A. D. S. Solteira, Estudo de Propriedades Físicas e Químicas de um novo Cimento Selador Endodôntico, Diss. Mestrado, Universidade Estadual Paulista, SP (2004).

[19] G. C. R. Garcia, E. M. B Santos, S. Ribeiro, Efeito do tempo de cura na rigidez de argamassas produzidas com cimento Portland, Cerâmica 57, 341 (2011) 94-99.

[20] A. B. Lima, O processo produtivo do cimento portland, Monografia (Especialização em Engenharia de Recursos Minerais), Universidade Federal de Minas Gerais, Belo Horizonte, MG (2011) 38p.

[21] E. Mastorakos, A. Massias, C. D. Tsakiroglou, D. A. Goussis, V. N. Burganos, A. C. Payatakes, CFD predictions for cement kilns including ame modelling, heat transfer and clinker chemistry, Appl. Math. Modelling 23 (1999) $55 \pm 76$ (1999) 55-76.

[22] Seminário de Operacíon de Hornos, Holderbank. Administracíon y Asesoría S.A. (1995) 292.

[23] H. A. Spang, A Dynamic Model of a Cement Kiln, Automatica 8, 6 (1972) 309-323.

[24] K. S. Mujumdara, V. V. Ranade, Simulation of rotary cement kilns using a one-dimensional model, Institution of Chemical Engineers, Chem. Eng. Res. Design (2006) 165177.

[25] A. L. de Castro, J. B. L. Liborio, V. C. Pandolfelli, A influência do tipo de cimento no desempenho de concretos avançados formulados a partir do método de dosagem computacional, Cerâmica 57, 341 (2011) 10-21.

[26] M. C. C. Silva, Relações entre microestrutura, composição, resistência à ruptura e moabilidade de clínqueres de cimento Portland, Diss. Mestrado, Engenharia Metalúrgica e de Materiais, COPPE/UFRJ, Rio de Janeiro, RJ (2007).

[27] L. G. Paula, Análise Termoeconômica do Processo de Produção de Cimento Portland com Co-Processamento de Misturas de Resíduos, Diss. Mestrado (em Conversão de Energia), Instituto de Engenharia Mecânica, Universidade Federal de Itajubá, MG (2009) 158p.

[28] P. Weber, "Heat Transfer in Rotary Kilns", ZementKalk-Gips, Bauverlag GMBH,Alemanha (1963).

[29] R. N. Shereve, J. A. Brink, "Indústrias de Processos Químicos", Ed. Guanabara Dois, $4^{\text {a }}$ Ed., Rio de Janeiro, RJ, (1980) 717.

[30] J. A. S. Tenório, F. G. S. Araújo, S. S. R. Pereira, A. V. Ferreira, D. C. R. Espinosa, A. Barros, Decomposição da fase majoritária do cimento Portland - Parte II: alita com adições de Fe e Al-Esc. Minas, Ouro Preto, MG (2003).

[31] Mineralogía Del Clinquer, Seminário de cimento Lima, Holderbank Administracíon y Asesoría S.A. (1979) 50.

[32] R. H. Perry, L. R. Benskow, W. E. Beimesch, "Perry's Chemical Engineers' Handbook", $8^{\text {a }}$ Ed., McGraw-Hill, N. York, EUA (2008).

[33] J. M. Smith, H. C. Van Ness, M. M. Abbott, "Introdução à Termodinâmica da Engenharia Química", Ed. LCT, $7^{\mathrm{a}}$ Ed. (2007).

[34] G. Kabir, A. I. Abubakar, U. A. El-Nafaty, Energy audit and conservation opportunities for pyroprocessing unit of a typical dry process cement plant, $U$. A. Energy 35, 3 (2010) 1237-1243.

(Rec. 10/07/2012, Rev. 10/12/2012, Ac. 15/12/2012) 